\section{Cahiers de Narratologie}

Analyse et théorie narratives

$40 \mid 2021$

École, Récits, Nation

\title{
Propos introductifs. École et Nation, co- construction et récits partagés
}

\section{Christel Taillibert et Nicole Biagioli}

\section{(2) OpenEdition}

1 Journals

Édition électronique

URL : https://journals.openedition.org/narratologie/12644

DOI : 10.4000/narratologie. 12644

ISSN : 1765-307X

Éditeur

LIRCES

\section{Référence électronique}

Christel Taillibert et Nicole Biagioli, « Propos introductifs. École et Nation, co-construction et récits partagés », Cahiers de Narratologie [En ligne], 40 | 2021, mis en ligne le 25 décembre 2021, consulté le 12 mai 2022. URL : http://journals.openedition.org/narratologie/12644 ; DOI : https://doi.org/10.4000/ narratologie. 12644

Ce document a été généré automatiquement le 12 mai 2022.

Article L.111-1 du Code de la propriété intellectuelle. 


\title{
Propos introductifs. École et Nation, co-construction et récits partagés
}

\author{
Christel Taillibert et Nicole Biagioli
}

1 Les textes rassemblés dans ce dossier des Cahiers de narratologie sont issus d'un séminaire de recherche franco-italien qui s'est déroulé à Nice (France) et à l'Aquila (Italie) entre février 2017 et novembre 2018. Intitulé École et éducation en France et Italie depuis la fin du XIXe jusqu'à Seconde Guerre mondiale, ce séminaire interdisciplinaire résultait de la collaboration entre le LIRCES (Laboratoire Interdisciplinaire Récits, Cultures et Société) et l'Università degli studi dell'Aquila. Il a été initié et organisé par Christel Taillibert et Marco Antonio D'Arcangeli, et a donné lieu à 23 communications, en langues française et italienne. Ce programme a bénéficié du label et du soutien financier de l'Université franco-italienne.

2 L'idée de ces rencontres, consistant à confronter et comparer le développement des systèmes scolaires et éducatifs, ainsi que les débats pédagogiques en Italie et en France, depuis la fin du XIXe siècle jusqu'à la Seconde Guerre mondiale, était issue du constat selon lequel un travail comparatif approfondi n'avait jamais été effectué jusqu'alors. Il s'agissait, donc, de combler une lacune pour le moins étonnante, en particulier au regard des importantes affinités et des liens substantiels qui unissent les deux pays d'un point de vue historique et culturel - et ceci de façon encore plus marquée dans la période considérée.

3 La présente publication résulte d'un recentrage de la problématique globale du séminaire autour de la rencontre entre trois concepts : École / Récit / Nation. Treize textes issus des interventions proposées lors des rencontres, signés par des chercheurs français et italiens, viennent nourrir cette réflexion croisée.

4 Pour explorer la façon dont s'articulent ces concepts dans une perspective comparative, nous avons conservé le point de départ historique que constitue l'éveil des nationalités au XIX ${ }^{e}$ siècle avec, d'une part, la brèche de la Porta Pia en 1870, qui met fin au pouvoir temporel de la Papauté et entérine le choix de Rome comme capitale du nouveau Royaume d'Italie et, d'autre part, l'avènement de la III $^{e}$ République en France, lequel constitue un tournant décisif dans la consolidation du projet républicain 
issu de la Révolution française. Nous avons maintenu comme point d'arrivée la Seconde Guerre mondiale, laquelle signe la fin de parenthèses totalitaires, plus ou moins longues, pour les deux pays (le Ventennio nero en Italie, le régime de Vichy en France), mais aussi le point de départ de la décolonisation ${ }^{1}$ qui va transformer le rapport entretenu au territoire national, à la nation, et au récit qui résulte de sa construction. Le rôle des intellectuels dans la construction de l'idée de "nation» est devenu prégnant au XVIII ${ }^{e}$ siècle, dans la lignée des Lumières, qui ont préparé les révolutions américaine et française, et formulé les principes égalitaires qui les ont inspirées. La construction des nations elles-mêmes repose sur des ingrédients que les historiens qui ont étudié la création de l'école publique en France, ont énoncé comme suit ${ }^{2}$ : une langue commune, une histoire commune (soit un scénario historique admis par tous), et des institutions gérées par un corps de fonctionnaires, parmi lesquelles l'école, chargée de la transmission unifiée des savoirs.

6 Si la nation est fondée sur le droit des peuples à disposer d'eux-mêmes - droit dont la mise à mal par le traité de Versailles en 1918 provoqua à terme le second conflit mondial -, la démocratie se donne pour mission d'assurer le respect et le bon fonctionnement de ce principe. Son système repose sur la séparation des pouvoirs exécutif, législatif et judiciaire et la représentation des citoyens par des élus responsables de leur mandat devant le peuple. L'école, à partir du moment où elle concerne tous les citoyens, devient ainsi le garant de la démocratie. Le droit à l'instruction de tous assure, en effet, l'égalité des citoyens et leur capacité à participer à la vie politique et à se sentir intégrés dans une même société. Ceci explique que chaque redressement d'un pays après une guerre s'accompagne d'une réforme structurelle du système scolaire.

7 En France, la défaite de 1871 fit ressentir la nécessité de créer une école publique pour restaurer l'image d'une nation affaiblie par la perte de l'Alsace-Lorraine, et créer un sentiment patriotique partagé. La III république succéda au $2^{\text {nd }}$ empire et les lois de Jules Ferry rendirent l'école gratuite (loi du 16 juin 1881), l'instruction primaire obligatoire et amorcèrent la laïcisation de l'enseignement public (loi du 28 mars 1882). La "question scolaire», qui dressait périodiquement les uns contre les autres les partisans de l'école publique et ceux de l'école religieuse, revint régulièrement sur le devant de la scène, jusqu'à ce que la défaite de 1940 induise le régime de Vichy, collaborationniste, à réformer l'éducation scolaire. L'enseignement primaire public fut alors réorienté vers un triple objectif, résumé par le triptyque suivant : "éducation morale, civique et patriotique, éducation intellectuelle, activités d'éducation générale à base d'éducation physique $»^{3}$. À la Libération, parmi les premières mesures prises par le gouvernement provisoire, figurèrent la refondation de l'école primaire et l'extension de la gratuité et de l'obligation scolaire à l'enseignement secondaire, à la fois pour restituer à l'éducation primaire sa mission d'instruction publique et pour former une nouvelle élite, la précédente s'étant largement compromise avec le régime de Vichy ${ }^{4}$.

En Italie, l'unification de la nation issue du Risorgimento entraîna immédiatement une grande campagne d'alphabétisation. L'école devint obligatoire et gratuite en 1878, mais eut les plus grandes difficultés à sortir du giron de l'Église. Plus tard, les désillusions provoquées par le traité de Versailles en 1918 suscitèrent la montée du fascisme qui remit l'école sous la tutelle de l'Église et l'orienta vers une préparation militaire destinée à soutenir les ambitions coloniales du régime ${ }^{5}$. La Seconde Guerre mondiale 
marqua à la fois la fin du fascisme, l'instauration de la république, et le retour à l'école publique laïque.

9 Ces mécanismes politiques, historiquement décalés mais tout à fait comparables entre la France et l'Italie, se caractérisent par leur façon paradoxale de faire de l'école à la fois la responsable des défaites et l'instrument des reconstructions nationales. Ils montrent que l'école est conçue dans la gestion des nations modernes comme un volant de régulation des crises provoquées tant par les dissensions internes que par les attaques externes. Une instrumentalisation qui se paie d'une absence de réflexion sur les spécificités de son fonctionnement, et explique que les sciences de l'éducation n'aient fait leur apparition qu'au début du $\mathrm{XX}^{\mathrm{e}}$ siècle.

Sur la base de ce cadre global, la réflexion développée dans ce numéro s'articulera autour de trois grandes parties, rendant successivement compte d'une réflexion sur la façon dont l'histoire de l'école s'articule avec les concepts d'État tout d'abord, d'éducation ensuite, et enfin de récit.

11 Dans la première partie, consacrée aux rapports de l'École et de l'État, sera exposée la façon dont la structuration des États français et italien conditionne la pensée même de l'école, dans une perspective éminemment idéologique. Marco Antonio D’Arcangeli montre ainsi, à travers l'évolution des théories éducatives exposées dans la Rivista pedagogica (1908-1939) - la principale revue consacrée à la pédagogie en Italie dans la première moitié $\mathrm{du} \mathrm{XX}^{\mathrm{e}}$ siècle -, comment la pensée bourgeoise, libérale et démocratique d'une part, qui inspire le processus de modernisation capitaliste du pays, et d'autre part la montée en puissance d'un sentiment de conscience nationale au sein des populations - sentiment tout nouveau dans le jeune État -, accompagnent les orientations théoriques et les propositions organisationnelles relatives à l'école au début du XXe siècle.

Parallèlement, en France, avec l'avènement de la III ${ }^{e}$ République, l'école pour tous devient le socle de la solidarité nationale. L'école laïque gratuite et obligatoire concorde avec les principes d'égalité et de fraternité, mais aussi avec la liberté, parce qu'elle met à la portée de tous des moyens autres que la force pour faire entendre sa voix : les capacités à échanger, à délibérer, à comprendre le fonctionnement de l'État. Les programmes scolaires vont, dans ce cadre, prendre en charge la construction d'un espace national. Ce phénomène est tout particulièrement prégnant dans le cadre de l'enseignement de la géographie. Comme le montre avec beaucoup d'acuité Jean-Pierre Chevalier dans son article, si cette discipline occupe une place importante dans le projet scolaire de la III ${ }^{e}$ République, c'est aussi parce qu'elle accompagne le projet de l'État dans la formulation de l'unité de la nation, sur un territoire remodelé par les colonies, au sein duquel est célébré l'efficacité de l'appareil institutionnel. La France, dans cette représentation partagée, apparait comme le centre du monde et le champion des valeurs universelles. Rappelons que l'institution scolaire telle qu'elle s'est développée dans l'empire colonial français rencontre des objectifs qui débordent ceux que l'on observe en métropole. En effet, la scolarisation devient le principal motif de justification de l'expansion coloniale ${ }^{6}$. L'État français se présente comme apportant la civilisation aux territoires de son empire. Il assure la formation des élites subalternes locales, et, ce faisant, prépare la transition vers la décolonisation qui débouchera sur la création d'États indépendants. Ceux-ci hériteront de l'ancienne métropole une langue véhiculaire, un appareil administratif, la maîtrise de l'écrit institutionnel et un réseau de relations internationales. Ce sont ces phénomènes qu'observe Nicole Biagioli dans 
l'article consacré à la méthode la plus connue d'enseignement du français dans les colonies : Mamadou et Bineta.

Il est frappant de constater à quel point la force du projet de société incarné par l'école de la III République repose sur la charge idéologique partagée par les acteurs de l'éducation. Les " Hussards noirs de la République " ${ }^{7}$ se sont employés à faire vivre, au sein de leurs classes, le projet républicain «à la française ». Même les franges les plus expérimentales de l'éducation participent de cette co-construction. Leslie Dagneaux montre, par exemple, comment les mouvements d'éducation nouvelle - et plus particulièrement le mouvement Freinet, objet de sa recherche - participent de ce projet global. Elle analyse en effet la façon dont, au sein de ce mouvement, le film, en tant qu'objet de création, est envisagé comme un outil d'émancipation culturelle et politique. Tout en s'ancrant profondément dans l'idéologie communiste et dans ses perspectives anticapitalistes, les propositions éducatives propres au mouvement Freinet s'attachent à permettre aux enfants de s'emparer des objets de culture et de construire leur rapport au monde en pleine autonomie. Ce processus d'émancipation s'accorde parfaitement avec les modes d'action développés à la même époque par l'éducation populaire qui, en marge de l'école, prolongeait son programme consistant, en droite ligne avec l'idéologie des Lumières, à lutter contre l'obscurantisme religieux, et à soustraire la science, la connaissance, la morale et la formation de la pensée à l'emprise dont bénéficiait à grande échelle la doctrine catholique. Comme le montre Pascal Laborderie, le travail réalisé sur le terrain, en France, par les réseaux laïques du cinéma éducatif, est parfaitement symptomatique de cette action concertée, grâce à laquelle l'État, conforté dans son action par un secteur associatif et/ou militant qui prolonge son action sous des formes diversifiées, structure son œuvre éducative autour de l'institution scolaire et de ses ramifications périscolaires.

La deuxième partie du volume est consacrée à un ensemble de réflexions sur la rencontre entre les concepts d'école et d'éducation. En effet, le développement de l'école introduit un nouvel espace dans la vie des citoyens, et par voie de conséquence l'instauration d'une rupture plus ou moins affirmée entre, d'une part, la vie privée, l'éducation reçue dans la famille et, d'autre part, la vie publique, professionnelle et citoyenne, dès lors que tous les citoyens sont appelés à participer, à un degré ou à un autre, à la prise de décision politique. Avec cette nouvelle vision de la citoyenneté, les sentiments d'appartenance qui s'ancraient majoritairement jusque-là dans la structure familiale et un environnement très local, vont trouver un terrain fécond dans une patrie assimilée à sa République, qui fait nation, y compris avec son empire colonial.

Comme nous l'avons suggéré précédemment, le premier obstacle à cette construction socio-psychologique était incarné, à l'époque qui nous intéresse, par la mainmise du pouvoir religieux sur l'éducation et, donc par l'influence qu'il exerçait sur les comportements politiques des citoyens. La question de la laïcité n'a pas eu, selon les époques, la même résonance dans les milieux éducatifs français et italien. En France, elle a constitué un objectif fondateur du combat mené pour consolider la démocratie, et le triptyque "école gratuite, obligatoire et laïque ", fait pendant à celui de la devise "liberté, égalité, fraternité ». Elle s'identifie à trois principes: la neutralité religieuse, la promotion de valeurs morales universelles et l'institutionnalisation de la démocratie ${ }^{8}$. La sécularisation de l'État est entérinée par la loi en 1905, mais ce combat va se poursuivre sur le terrain de l'éducation jusqu'à la Seconde Guerre mondiale, et audelà. En Italie aussi, la question de la laïcité était au cœur des débats qui ont agité la 
sphère sociale et politique après le Risorgimento. Carmen Betti, dans son article, analyse la façon dont la question de l'enseignement religieux à l'école a constitué un espace de confrontation entre les différentes forces politiques en présence, et ce jusqu'à ce que la réforme de l'éducation menée par Giovanni Gentile en 1923 réintroduise l'enseignement religieux obligatoire dans les écoles du royaume ${ }^{9}$, puis que soient signés, entre l'Église catholique et l'État, les accords du Latran en 1929. Résultant d'une manœuvre diplomatique de Benito Mussolini, ces textes confirmaient le statut de religion d'État au catholicisme, mais réduisaient la souveraineté du pape au seul État du Vatican. Ajoutons que, dans les deux pays, la longévité de l'école libre s'explique aussi par un facteur socioculturel étranger à la conviction religieuse : préserver les enfants, en particulier les filles, des mauvaises fréquentations et maintenir les barrières sociales compromises par l'école laïque.

Toutefois, il ne faudrait pas oublier que, pendant très longtemps, l'Église fut le seul acteur de l'éducation, et que ce sont les ordres religieux qui ont pensé et diffusé les premiers modèles pédagogiques, des plus conformistes aux plus novateurs. Il en reste des traces dans le silence observé quand le maître parle et dans l'immobilisation des corps en class $^{10}$, mais également dans l'exercice de la parole publique et l'enseignement de la rhétorique, du latin et de la philosophie. Ainsi, le corps - comme l'esprit - constitue un pivot de l'éducation laïcisée : l'école met en place une éducation du corps par l'exercice, l'hygiène et la santé. Gaetano Bonetta observe dans son article la façon dont se transforme, au cours du XIX ${ }^{\mathrm{e}}$ siècle, le regard porté sur le corps, et comment ces transformations accompagnent le développement de la gymnastique, envisagée comme un outil de préservation du corps humain. Ces profondes mutations culturelles préparent par ailleurs la voie à l'exaltation de la force physique qui soustendra la militarisation de l'éducation et l'encadrement de la jeunesse dans l'Italie mussolinienne, et la création des « chantiers de jeunesse » dans la France de Vichy.

17 La question de la place réservée aux femmes dans la société est prégnante dans la réflexion relative aux relations entre l'État et l'éducation. Traditionnellement, seules les classes sociales privilégiées - noblesse, haute bourgeoisie - pouvaient assurer par choix personnel à leurs filles un véritable enseignement, par préceptorat, la majorité préférant les confier à des couvents ou des institutions religieuses où l'éducation morale et l'acquisition de quelques talents de société prévalaient sur la formation proprement scolaire ${ }^{11}$. Dans les autres classes sociales, la question de l'éducation des filles a toujours été envisagée de façon très différente de celle des hommes, et ce en raison même du rôle qui leur était attribué dans la société : entretenir le foyer, éduquer les enfants, tandis que l'homme est appelé à travailler à l'extérieur pour subvenir aux besoins de la famille. D'où la séparation que l'on observe au sein de l'école publique : si une éducation de base doit être apportée aux filles comme aux garçons, cette discrimination interdira longtemps aux filles l'accès aux études secondaires, puis supérieures, aux matières qui y préparent (latin et philosophie dans le modèle des études humanistes) et aux carrières qui en dépendent. Plusieurs articles dans ce numéro explorent la place des femmes dans la société et dans l'environnement éducatif global. Revenant sur les fondements révolutionnaires de la pensée éducative qui marque le modèle républicain français, Antonia Criscenti analyse le parcours de deux femmes engagées sur le terrain des droits des femmes et de l'éducation: Sophie de Grouchy, qui introduit la question de la sensibilité en tant que variable clef de l'action pédagogique, et Olympe de Gouges, qui associe étroitement sa réflexion pédagogique à 
un militantisme politique et social en faveur du droit des femmes, ce qui lui vaudra, dans le contexte de la Terreur, d'être condamnée à mort.

Gabriele Armenise s'intéresse à une autre figure féminine, Anna Vertua Gentile. Au tournant du $\mathrm{XX}^{\mathrm{e}}$ siècle, celle-ci rédigea plusieurs manuels destinés aux femmes, afin de leur transmettre un certain nombre de préceptes relatifs aux « bons » comportements à adopter au sein de la société. Ces ouvrages constituent aujourd'hui une grille de lecture efficace pour comprendre le rôle dévolu à l'éducation à une époque où elle était envisagée comme un instrument visant à l'acquisition d'un «code», d'une " étiquette ", bref, un outil de formatage des comportements - et particulièrement, donc, des comportements féminins.

Enfin, la réflexion menée sur la place des femmes dans l'éducation se construit dans ce volume autour d'une ultime figure féminine, Simone Weil. Elle est à elle seule un symbole des acquis des femmes en matière d'éducation. En effet, les lois scolaires de la fin du XIX ${ }^{e}$ siècle interdisent aux filles non seulement les disciplines scientifiques mais aussi le latin et la philosophie ${ }^{12}$. L'enseignement secondaire, qui forme les élites, leur est ouvert seulement en 1880 par la loi Camille Sée, et il est différencié de celui des garçons, notamment par l'absence du latin et de la philosophie. Il faut attendre 1924 pour que l'instauration d'un baccalauréat unique pour les deux sexes leur ouvre les portes de l'université, et 1937 pour que les femmes soient autorisées à enseigner le latin, le grec et la philosophie.

Professeur de philosophie, Simone Weil est aussi et surtout une philosophe dont la pensée originale a marqué son époque. Sa philosophie de l'éducation s'est élaborée à partir de la rencontre entre une pratique de l'enseignement dans les lycées de filles, et une expérience d'ouvrière en usine. Elle s'articule autour de la problématique récurrente de la " mise en action » et l'a conduite à s'engager dans la France Libre, aux côtés du général de Gaulle à Londres.

Comme en témoignent les différentes problématiques soulevées par les auteurs participant à ce volume, l'éducation, si elle s'enracine dans une institution scolaire pensée par l'État éducateur, trouve des prolongements dans la société tout entière et dans tous les espaces de la vie - publique et privée. Elle résulte à la fois des choix politiques, d'une philosophie de l'éducation et d'une orientation conférée au corps social dans son ensemble. D'où l'idée, qui structurera la troisième et ultime partie de ce dossier, selon laquelle l'éducation est profondément et structurellement liée à l'idéologie sous-jacente à l'ordre social qui l'institue. Cette proposition nous amène ainsi à envisager l'œuvre éducative comme participant d'un "récit» national, coordonné par l'État, et qui trouve des prolongements narratifs dans chaque œuvre éducative dispensée dans son cadre. Ce paradigme est évident dès lors que l'on se penche sur les formes éducatives qui adoptent, fort à propos, une structure narrative. C'est selon cette perspective que Nicole Biagioli compare les tenants et les aboutissants de deux romans éducatifs, le Tour de la France par deux enfants de Giordano Bruno (1877) en France, et sa version « fascisée » signée par Giacomo Cavallucci et Jack H. Rousset en 1935, Le tour de France de Mimmo et Mammola. Mais il se vérifie aussi, de façon plus globale, dans la façon dont le "récit national», fondateur de l'idéologie partagée, trouve des échos dans l'œuvre éducative tout entière, au niveau structurel comme au niveau de ses contenus. C'est sur ce phénomène que se penchent les deux derniers textes de ce volume. Celui de Stefano Lentini réfléchit, dans le cadre du Ventennio fasciste, à la façon dont l'instruction artistique ainsi que l'éducation esthétique ont été 
mises au service de l'idéologie d'État et de sa divulgation auprès du peuple. Celui de Christel Taillibert adopte, quant à lui, une perspective comparative et, se concentrant sur la question de la cinématographie éducative dans chacun des deux pays considérés, analyse la façon dont ces environnements, au sein et en dehors de l'école, peuvent être pensés comme des rouages au service de l'écriture et de la diffusion du « récit national ».

Ce sont donc trois types de récits qui ont été envisagés : le récit national qui forge l'inconscient culturel partagé par une majorité de citoyens; le récit de l'histoire enseignée en classe qui assure la transmission d'une mémoire nationale exacte mais forcément sélective; et le récit de l'histoire historienne, critique, théorisé et comparatif. Ces trois types, nous l'avons vu, sont souvent intriqués. Les films de propagande et les films d'actualité sont programmés dans les mêmes séances, les tours de la patrie sont distribués gratuitement dans les écoles; et faire commencer l'histoire de la France à la Gaule ou l'histoire de l'Italie à Rome, est un indice clair de l'intrusion $\mathrm{du}$ récit national dans la chronologie historique. Cela pose forcément un problème d'identification aux populations émigrées récentes, d'où la nécessité d'intégrer les passés des individus dans celui de la nation.

Mais ces trois types de récit sont aussi interdépendants, et mieux connaître leurs relations permet de comprendre comment chacun s'insère dans le mécanisme global qui génère l'opinion publique. C'est donc sur le principal moyen d'y parvenir : l'histoire comparée $^{13}$, que nous souhaitons clore ce prologue. Deux histoires nationales ne font pas la vérité historique mais peuvent y contribuer, notamment quand les pays sont limitrophes, ce qui induit de fortes interactions: influences, guerres, alliances, ainsi que des cultures et des histoires partagées qui font ressortir les différences de ponctuation historique. Axer la réflexion historienne sur l'histoire partagée est donc un moyen sûr de décentrer le point de vue nationaliste qui organise le récit national. Nous y ajouterons une autre clause : choisir l'éducation comme axe de comparaison est une stratégie particulièrement fiable si l'on souhaite analyser sur le temps long les rouages de la fabrique citoyenne, et la façon dont chacun y participe, comme acteur et comme décideur.

\section{BIBLIOGRAPHIE}

Barthélémy, Pascale. «L'enseignement dans l'Empire colonial français : une vieille histoire ? ", Histoire de l'éducation, n¹08, oct-déc. 2010, pp. 5-28.

Boucheron, Patrick ; Delalande, Nicolas. Pour une histoire-monde. Paris, Puf, 2013.

Cavel, Isabelle, «Réformer l'école après 1944 : du consensus au dissensus entre la SFIO et le MRP », Histoire @Politique, n¹8, septembre-décembre 2012 [en ligne] https://www.histoirepolitique.fr/index.php?numero=18\&rub=autres-articles\&item $=70$

Coq, Guy. «La laïcité et l'école publique », Trema [En ligne], 37 | 2012, mis en ligne le 01 avril 2014, consulté le 13 juin 2017 http://trema.revues.org/2747 ; DOI : 10.4000/trema.2747 
Foucault, Michel. Surveiller et punir. Paris, Gallimard,1975.

Greenwalt, Kyle A. "Discourse, narrative, and National Identity: The Case of France", Harvard Educational Review, vol. 17, n³, 2009, pp. 494-520.

Mayeur, Françoise. « Garçons et filles du XIX au XX siècle : une éducation différente ", Enfance, $1981, \mathrm{n}^{\circ} 1$, pp. 153-154.

Ostenc, Michel. «L'école italienne pendant le Fascisme », Revue d'histoire moderne et contemporaine, $\mathrm{n}^{\circ} 3$, juillet-septembre 1983, pp. 401-407.

Veyne, Paul. Comment on écrit l'histoire. Seuil, 1979.

\section{NOTES}

1. Du fait de leur expansion coloniale, les deux pays font partie de ce que les historiens appellent des États-empires par opposition aux États-nations, l'empire étant « une grande entité politique, expansionniste ou ayant une mémoire de l'expansion, maintenant les distinctions et les hiérarchies, tout en incorporant de nouvelles populations » (Burbank, Jane ; Cooper, Frederick. « Un monde d'empires », in Boucheron, Patrick ; Delalande, Nicolas. Pour une histoire-monde. Paris, Puf, 2013, p. 39).

2. Voir par exemple : Greenwalt, Kyle A. "Discourse, narrative, and National Identity: The Case of France", Harvard Educational Review, vol. 17, n³, 2009, pp. 494-520.

3. "Arrêté du 16 août 1941 relatif à l'organisation de l'enseignement primaire élémentaire ", article 17. Publié au Journal Officiel de l'État français, 2 septembre 1941, n² 244, p. 3710.

4. Cavel, Isabelle, «Réformer l'école après 1944 : du consensus au dissenssus entre la SFIO et le MRP », Histoire @Politique, n¹8, septembre-décembre 2012 [en ligne]

https://www.histoire-politique.fr/index.php?numero=18\&rub=autres-articles\&item=70

5. Vu le décalage historique de l'expansion coloniale de l'Italie par rapport à la France, on ne s'étonnera pas que l'école y ait d'abord été chargée de former les colonisateurs, avant les colonisés (Voir : Vino, Massimiliano. Educare all'Impero, Apprendimento coloniale all' Italia fascista, 27 giugno 2019: «A pochi mesi dalla proclamazione dell'Impero, il ministro dell'Educazione Giuseppe Bottai emanò una circolare sollecitando l'introduzione della dimensione imperiale nell'insegnamento. Nel 1936 al libro unico della classe quinta elementare fu aggiunto ad esempio un capitolo dedicato esclusivamente alla conquista dell'Etiopia» (Nous traduisons : Éduquer à l'Empire, apprentissage colonial à l'Italie fasciste : «À quelques mois de la proclamation de l'Empire, le ministre de l'Éducation Giuseppe Bottai publia une circulaire exigeant l'introduction de la dimension impériale dans l'enseignement. En 1936, on ajouta au livre unique de cinquième année de l'école élémentaire un chapitre exclusivement dédié à la conquête de l'Éthiopie »).

https://www.treccani.it/magazine/chiasmo/storia_e_filosofia/Apprendimento/

SSSGL_Educare_impero.htm

6. À preuve cette réflexion d'Albert Sarraut, ministre des colonies: «Ce problème de l'enseignement est sans doute le plus important et le plus complexe de ceux qui sollicitent l'esprit du colonisateur, car il contient plus ou moins en puissance tous les autres et affectent leur solution " (Sarraut, Albert. Grandeur et servitude coloniales, Paris, Éd. du Sagittaire, 1931, p. 146, cité par Barthélémy, Pascale. «L'enseignement dans l'Empire colonial français : une vieille histoire ?", Histoire de l'éducation, n 108, oct-déc. 2010, p. 5).

7. Expression créée par Charles Péguy, en 1913, inspirée par l'uniforme des instituteurs : une redingote et un pantalon noir, et par leur patriotisme militant.

8. Voir : Coq, Guy. «La laïcité et l'école publique », Trema [En ligne], 37 | 2012, mis en ligne le 01 avril 2014, consulté le 13 juin 2017.URL : http://trema.revues.org/2747 ; DOI : 10.4000/trema.2747 
9. Voir : Ostenc, Michel. "L'école italienne pendant le Fascisme », Revue d'histoire moderne et contemporaine, $\mathrm{n}^{\circ} 3$, juillet-septembre 1983, pp. 401-407.

10. Voir : Foucault, Michel. Surveiller et punir. Paris, Gallimard,1975, section III, chapitre premier : Les corps dociles, pp. 147-151. Foucault y décrit l'organisation des collèges jésuites sur le modèle guerrier de la légion romaine (deux ou trois cents élèves divisés en groupes de dix, répartis en deux légions, la romaine et la carthaginoise, qui s'affrontent par résultats scolaires interposés) ; et l'organisation sérielle de l'espace scolaire par Jean-Baptiste de la Salle, fondateur des Frères des écoles chrétiennes, qui corrèle la place de l'élève aux : niveau scolaire, sujet à étudier, rang social (indicatif du niveau d'hygiène), tempérament, et moralité.

11. La loi Falloux (1850), qui est la première loi à s'intéresser spécifiquement à l'éducation des filles « donne en la matière des privilèges tels aux congrégations enseignantes qu'elle aboutit pratiquement à mettre l'instruction des filles entre les mains de religieuses » (Mayeur, Françoise. "Garçons et filles du XIX au XX ${ }^{\mathrm{e}}$ siècle : une éducation différente », Enfance, 1981, n¹, p. 45).

12. En effet, «L'explication se trouve sans doute dans la préoccupation de ne pas mettre les filles, si peu que ce soit, en concurrence avec les garçons. Le latin est alors le fondement de l'enseignement secondaire [...]. La philosophie est le couronnement de cette instruction » (Ibid., p. 49).

13. Comme le remarque Paul Veyne: «L'étude de n'importe quelle civilisation enrichit la connaissance que nous avons d'une autre [...] la recette est à peu près infaillible pour renouveler n'importe quel point d'histoire et les mots d'étude comparée devraient être au moins aussi consacrés que ceux de bibliographie exhaustive » (Veyne, Paul. Comment on écrit l'histoire. Seuil, 1979, p. 17). 